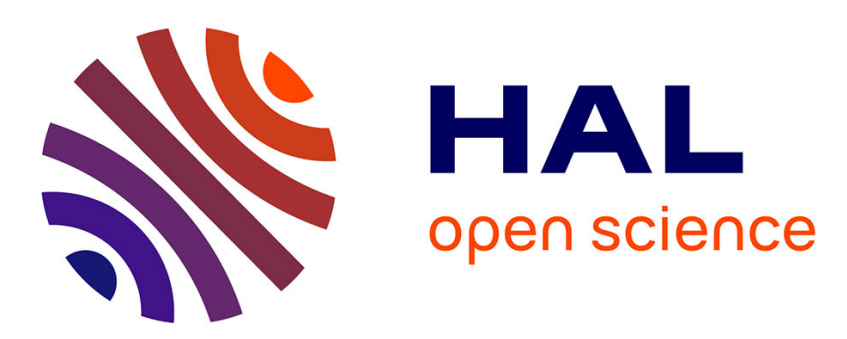

\title{
LES SOURCES D'INEFFICACITÉ DES MÉCANISMES DE GOUVERNANCE D'ENTREPRISE
}

Sandrine Boulerne, Jean-Michel Sahut

\section{> To cite this version:}

Sandrine Boulerne, Jean-Michel Sahut. LES SOURCES D'INEFFICACITÉ DES MÉCANISMES DE GOUVERNANCE D'ENTREPRISE. Revue management \& avenir, 2010, 10.3917/mav.033.0374 . halshs-02099278

\section{HAL Id: halshs-02099278 \\ https://shs.hal.science/halshs-02099278}

Submitted on 23 Apr 2019

HAL is a multi-disciplinary open access archive for the deposit and dissemination of scientific research documents, whether they are published or not. The documents may come from teaching and research institutions in France or abroad, or from public or private research centers.
L'archive ouverte pluridisciplinaire $\mathbf{H A L}$, est destinée au dépôt et à la diffusion de documents scientifiques de niveau recherche, publiés ou non, émanant des établissements d'enseignement et de recherche français ou étrangers, des laboratoires publics ou privés. 


\title{
LES SOURCES D'INEFFICACITÉ DES MÉCANISMES DE GOUVERNANCE D'ENTREPRISE
}

Sandrine Boulerne et Jean-Michel Sahut

\author{
Management Prospective Ed. | « Management \& Avenir »
}

2010/3 n³3 | pages 374 à 387

ISSN 1768-5958

Article disponible en ligne à l'adresse :

https://www.cairn.info/revue-management-et-avenir-2010-3-page-374.htm

Distribution électronique Cairn.info pour Management Prospective Ed..

(C) Management Prospective Ed.. Tous droits réservés pour tous pays.

La reproduction ou représentation de cet article, notamment par photocopie, n'est autorisée que dans les limites des conditions générales d'utilisation du site ou, le cas échéant, des conditions générales de la licence souscrite par votre établissement. Toute autre reproduction ou représentation, en tout ou partie, sous quelque forme et de quelque manière que ce soit, est interdite sauf accord préalable et écrit de l'éditeur, en dehors des cas prévus par la législation en vigueur en France. Il est précisé que son stockage dans une base de données est également interdit. 


\title{
Les sources d'inefficacité des mécanismes de gouvernance d'entreprise
}

\author{
par Sandrine Boulerne ${ }^{122}$ et Jean-Michel Sahut ${ }^{123}$
}

Résumé

Les derniers scandales révélés en pleine crise des subprimes montrent que ces mécanismes de gouvernance ne fonctionnent que de façon imparfaite. En effet, on constate que la plupart des entreprises ne font pas de la gouvernance un enjeu majeur. Elles sont plutôt dans une démarche de respect formel de dispositions réglementaires ou professionnelles plutôt que dans celle d'une adhésion réelle aux principes de gouvernance. Dans ce contexte, cet article a pour principal objectif d'identifier les mécanismes de gouvernance applicables aux sociétés par action et d'expliquer leurs sources d'inefficacité en France. Outre les causes culturelles, organisationnelles et environnementales, les auteurs avancent comme explication principale que les dirigeants considèrent que les mécanismes de gouvernance concernent plus la non-destruction qu'une véritable création de valeur.

Abstract

The subprime crisis showed that governance mechanisms are still far from perfect in most organisations. Indeed, the majority of organisations do not appear to consider governance as a key issue. Instead, they work from the standpoint of formal respect for the regulatory or professional provisions in place rather than one of real adherence to governance principles. In this context, the article's main objective is to identify the mechanisms of governance applicable to companies and explain their sources of inefficiency in France. We believe that, in addition to cultural, organisational and environmental issues, the main explanation is that executives consider governance mechanisms to be more to do with the non-destruction of value rather than with real value creation.

La dissociation entre la propriété et le contrôle dans les sociétés par actions est à l'origine de l'émergence du concept de gouvernance. Selon la théorie de l'agence, telle qu'élaborée par Jensen et Meckling (1976), cette dissociation crée un problème de conflits d'intérêts potentiels notamment entre les actionnaires et les dirigeants. Plus la propriété est disséminée entre un grand nombre d'actionnaires, plus les dirigeants risquent de gérer l'entreprise dans leur propre intérêt. En effet, les dirigeants ont des objectifs et des horizons temporels différents de ceux des actionnaires et disposent de l'accès privilégié à l'information qu'ils peuvent mettre

122. Sandrine Boulerne, ESCEM et maître de conférences à l'IAE de TOURS, SBOULERNE@escem.fr

123. Jean-Michel Sahut Groupe Sup de Co Amiens, chercheur associé au Cerege, Université de Poitiers, JeanMichel.SAHUT@ supco-amiens.fr 


\section{Les sources d'inefficacité des mécanismes de gouvernance d'entreprise}

à profit en orientant la gestion de l'entreprise vers la réalisation de leur objectif personnel. Par ailleurs, les dirigeants sont aussi capables de favoriser certains investissements par rapport à d'autres, en fonction de leur préférence et de leur risque. (Charreaux, 1991). Ainsi, la mise en place de mécanismes de gouvernance efficace devrait réduire les coûts associés aux conflits d'agence, assurer l'alignement des intérêts entre les actionnaires et les dirigeants et maximiser la richesse des actionnaires ${ }^{124}$. Dans cette optique, la gouvernance des sociétés peut se définir comme étant « l'ensemble des mécanismes organisationnels et institutionnels (dont les lois) qui délimitent l'espace discrétionnaire du dirigeant et influencent leur prise de décision »(Charreaux, 1997).

Les scandales financiers au niveau international des années 2000 (Enron, Worldcom, Parmalat) et la perte de confiance résultante des investisseurs envers la gestion des firmes ont conduit à un renforcement des mécanismes internes et externes de gouvernance notamment au niveau législatif (loi SarbanesOxley aux États-Unis ou la loi TEPA en France) et des règles « de bonne gouvernance » édictées ou proposées par divers organismes réglementaires ou professionnels (principes de l'OCDE, rapport Dey au Canada, rapport Cadbury en Grande-Bretagne, rapport Treadway aux Etats-Unis, etc.), sans oublier les pressions exercées par les investisseurs institutionnels. Outre leur portée éthique, l'idée centrale de ces mesures est qu'une « bonne gouvernance » est créatrice de valeur. Selon McKinsey (2002), les investisseurs institutionnels sont prêts à payer une prime de 12 à $14 \%$ pour les entreprises qui adoptent les meilleures pratiques de gouvernance. Ce lien a d'ailleurs été démontré par de nombreuses études qu'elles se soient intéressées à la gouvernance en générale ou à des mécanismes particuliers comme la structure de propriété, le rôle des investisseurs institutionnels, la proportion des directeurs externes dans le conseil d'administration, la présence d'une structure de dualité dans le conseil d'administration, ou encore la rémunération des dirigeants.

Toutefois, les derniers scandales révélés en pleine crise des subprimes montrent que ces mécanismes de gouvernance ne fonctionnent que de façon imparfaite. En effet, on constate que la plupart des entreprises ne font pas de la gouvernance un enjeu majeur. Elles sont plutôt dans une démarche de respect formel de dispositions réglementaires ou professionnelles plutôt que dans celle d'une adhésion réelle aux principes de gouvernance. On peut donc légitimement s'interroger sur les raisons expliquant cette situation.

Dans ce contexte, cet article a pour principal objectif d'identifier les mécanismes de gouvernance applicables aux sociétés par action et d'expliquer leurs sources d'inefficacité en France. Outre les causes culturelles, organisationnelles et environnementales, les auteurs avancent comme explication principale que les

124. Cet article adopte la vision traditionnelle juridico-financière de la gouvernance et du rôle des actionnaires et n'aborde pas la question du rôle cognitif des actionnaires. 


\section{3 \\ management \& avenir}

dirigeants considèrent que les mécanismes de gouvernance concernent plus la non-destruction qu'une véritable création de valeur.

\section{Les mécanismes de gouvernance}

\subsection{Les réponses institutionnelles aux scandales financiers}

Les scandales financiers et comptables à répétition et l'effondrement boursier de plusieurs sociétés ces dernières années (Enron en 2001 ; Andersen, Worldcom et Vivendi en 2002 ; Parmalat en 2003 ; Elf et Crédit Lyonnais en 2004), ont crée un véritable traumatisme au plan international. En réaction à cette série de scandales financiers, les législateurs ont été amenés à légiférer pour restaurer la confiance des investisseurs. C'est le cas de la Loi Sarbanes-Oxley (SOX) de juillet 2002 aux Etats-Unis ou de la Loi sur la Sécurité Financière (LSF) d'août 2003 en France. Ces deux lois imposent de nouvelles obligations en matière de contrôle interne. En France, la LSF traduit une volonté forte du législateur d'améliorer la transparence financière des firmes cotées en obligeant ces entreprises à présenter à l'assemblée générale, l'organisation des travaux du conseil d'administration, les procédures de contrôle interne et les délégations de pouvoirs. La loi Breton du 26 juillet 2005 et la loi TEPA du 21 août 2007 concernent davantage l'encadrement des engagements indemnitaires des mandataires sociaux (président du conseil d'administration, directeur général, directeur général délégué et membre du directoire) de sociétés cotées, en les soumettant à des critères de performance. Dans un contexte de crise financière, le législateur a encadré récemment les conditions de rémunération des dirigeants des entreprises aidées par l'Etat (décret du 30 mars 2009).

Outre les lois, plusieurs rapports dont le premier rapport Viénot de 1995 ont eu pour objectif d'émettre des recommandations sur la gouvernance des entreprises, en particulier pour renforcer l'indépendance des administrateurs et contrôler les décisions du président du conseil d'administration. Le mouvement des entreprises françaises (MEDEF) a également proposé des recommandations en octobre 2008 relatives à la rémunération des mandataires, y incluant les « parachutes dorés », afin que les entreprises mettent rapidement en œuvre des pratiques d'indemnités conformes à la bonne gouvernance ${ }^{125}$. Face aux récentes « révélations » sur les salaires des dirigeants, et autre bonus, stock-options et retraites-chapeaux, la présidente du MEDEF a proposé la création, avant fin avril 2009, d'un « comité des sages » pour surveiller les rémunérations des grands patrons. L'objectif de ce comité est de veiller à ce que les dirigeants mandataires sociaux des entreprises mettant en œuvre un plan social ou recourant massivement au chômage partiel reconsidèrent l'ensemble de leur rémunération. 


\section{Les sources d'inefficacité des mécanismes de gouvernance d'entreprise}

L'importance accordée aux mécanismes de gouvernement d'entreprise s'est également traduite par des initiatives multiples, visant à expliquer les « meilleures pratiques » de gouvernance. Ces initiatives se sont traduites par un ensemble de « codes de bonne conduite » destiné aux dirigeants d'entreprise, contenant des recommandations se voulant comme un « idéal » à atteindre ${ }^{126}$. D'ailleurs, Aguilera et Cuervo-Cazurra (2004) démontrent une croissance quasi-exponentielle des codes de gouvernance à partir du milieu des années 1990, le premier étant le rapport britannique Cadbury en 1992, suivi du « UK combined code on Corporate Gorvernance » en 2003. En France, l'AFEP'27 et le MEDEF ont également publié, en octobre 2003, un document intitulé « Principes de gouvernement d'entreprise $»^{128}$. Ces initiatives concernent essentiellement la dissociation des fonctions de président et de directeur général, les caractéristiques des administrateurs, la composition et le fonctionnement du conseil d'administration et la qualité de l'information financière et comptables.

En tant que mécanisme de gouvernement externe, les investisseurs institutionnels peuvent également contraindre les dirigeants à améliorer leur publication financière. Ils demandent plus de transparence, d'éthique et de comparabilité entre les entreprises. A titre d'exemple, les investisseurs institutionnels anglosaxons ont largement influencé l'adoption des comités de surveillance par les entreprises françaises cotées, en raison de leur participation grandissante dans le capital de ces firmes françaises (Thierry-Dubuisson, 2002).

\subsection{Les grandes tendances et réalité des pratiques de gouvernance}

Depuis ces dix dernières années, les pressions, notamment institutionnelles et légales, auraient fait évoluer la gouvernance des firmes vers un rôle accru des administrateurs, un développement des comités de surveillance et une séparation des pouvoirs. En effet, suite aux pressions des investisseurs institutionnels et des actionnaires minoritaires, le statut d'administrateur indépendant est apparu en 2002 (rapport Bouton) afin d'assurer une plus grande indépendance du conseil d'administration, au-delà des points de vue des dirigeants ou des actionnaires majoritaires. Les recommandations et bonnes pratiques font qu'en général le pourcentage d'administrateurs indépendants dans un conseil d'administration de société cotée est de l'ordre d'un tiers. La pratique a largement rejoint les recommandations des codes de « bonne gouvernance » dans toutes les grandes sociétés.

Outre l'indépendance des administrateurs, la création de comités de surveillance au sein du conseil d'administration des entreprises françaises cotées a également fait l'objet de multiples pressions. Le premier rapport Viénot (1995) a introduit une 
recommandation relative à la mise en place de comités de surveillance, plus particulièrement de comités d'audit, de nomination et de rémunération. Depuis la publication de ce rapport, la pression exercée par les investisseurs institutionnels anglo-saxons sur les entreprises cotées pour qu'elles s'alignent sur les standards de « bonne gouvernance » s'est accrue et les exigences se sont renforcées, notamment en ce qui concerne le nombre d'administrateurs indépendants qui composent ces comités. La mise en fonction de ces comités répond aussi aux pressions des politiques et de l'opinion publique suite aux récents scandales relatifs aux rémunérations excessives des dirigeants et à la «manipulation» des comptes.

La rémunération des dirigeants fait également l'objet de pressions, voire d'une vive controverse, de la part de nombreux actionnaires, notamment en assemblée générale. Cette dernière décennie, la part des plans d'actionnariat et d'options sur action (stock-options) s'est considérablement accrue dans la rémunération des dirigeants ${ }^{129}$. Outre le caractère incitatif des rémunérations, les dirigeants qui possèdent des parts dans l'entreprise supportent les conséquences des décisions nuisibles à l'entreprise et profitent de celles qui en augmentent la valeur. II s'ensuit que les dirigeants ayant une fraction de propriété plus importance fourniraient davantage d'efforts, auraient de plus longs horizons d'investissement et prendraient de meilleures décisions d'investissement. Si l'actionnariat des dirigeants est reconnu comme l'une des méthodes la plus efficace et la plus directe pour aligner les intérêts des dirigeants avec ceux des actionnaires, on voit se développer l'opposition de nombreux actionnaires, compte tenu des montants en jeu. Les conseils d'administration sont donc soumis à une pression croissante des actionnaires qui veulent s'assurer qu'il existe une relation effective entre l'octroi de telles formules de rémunération et la performance financière spécifique de la firme ${ }^{130}$. En outre, les contestations dépassent largement les actionnaires et les investisseurs institutionnels pour impliquer d'autres parties prenantes comme les salariés et leurs syndicats, les milieux politiques et les médias. De même, dans l'opinion publique, les stock-options alimentent les critiques. Certains sondages indiquent que les trois quarts des français se plaignent de la rémunération excessive des dirigeants et estiment que les chefs d'entreprise n'ont pas à bénéficier de tels compléments de rémunération ${ }^{131}$. II ne s'agit plus seulement d'aborder la question du lien entre rémunération et performance

\footnotetext{
129. A titre d'exemple, Antoine Zacharias, patron de Vinci, percevait un salaire de 4,3 millions d'euros, une prime de départ de 13 millions, une retraite de 2,2 millions et des stock-options pour un montant évalué à 173 millions. Lorsqu'il a réclamé un petit bonus supplémentaire de 8 millions d'euros, il fut contraint à la démission par son conseil d'administration le $1^{\text {er }}$ juin 2006 . Noël Forgeard démissionnait d'EADS avec l'équivalent de trois ans de salaire, à savoir 6,6 millions d'euros, en juillet 2006. Malgré la crise, l'année 2008 ne déroge pas à la règle. Franck Riboud, pdg du groupe Danone, Bernard Arnault, pdg de LVMH, Jean-Paul Agon, pdg de l'Oréal, restent les trois patrons les mieux rémunérés de France, selon l'enquête du magazine Les Echos. Mais seul le premier voit son salaire fixe et variable progresser de $7 \%$ à 4,28 millions d'euros. Les deux autres, crise oblige, n'ont respectivement perçu que 3,9 (-3\%) et 3,5 (-13\%) millions d'euros. Patrick Kron, d'Alstom, a perçu 18,8 millions d'euros dont 14,4 millions de stock-options pour l'année 2008.

130. Selon le journal Le Point (30 avril 2009), la baisse des résultats des entreprises en 2008 s'est répercutée sur la moitié des rémunérations des dirigeants des sociétés du CAC 40. En effet, Michel Rollier, patron de Michelin (2,5 millions d'euros) ou encore Carlos Ghosn (1,2 million d'euros), ont perdu chacun $54 \%$ de leur salaire.

131. Les indemnités de départ du président de Valéo et de celui de Dexia ont montré que les dirigeants doivent désormais compter avec l'opinion publique.
} 


\section{Les sources d'inefficacité des mécanismes de gouvernance d'entreprise}

mais aussi de s'interroger sur l'éthique du système de rémunération qu'ils sont chargés de concevoir et de gérer. Indépendamment de leur rémunération, les dirigeants ont également vu leur statut évolué. En France, la dissociation des fonctions de Président et de Directeur Général était interdite par la loi dans la société anonyme ${ }^{132}$. Mais l'explosion du nombre de scandales dans les années 90 dus pour beaucoup à des abus de pouvoirs de certains dirigeants a généré certaines tensions à l'origine de la loi NRE en 2003. Cette loi introduit dorénavant la faculté de séparer les rôles entre Président et Directeur Général au sein des sociétés cotées françaises et aujourd'hui plus de 51\% des sociétés du SBF 120 ont adopté cette séparation (Stuart, 2007).

Selon l'étude de Deloitte (2006), un certain nombre d'administrateurs reconnaissent explicitement que malgré le cadre plus contraignant, les nouvelles règles de gouvernance d'entreprise et la nouvelle culture d'entreprise mènent à une plus grande transparence. Toutefois, l'adoption des « pratiques de bonne gouvernance » comporte un réel danger, la pratique de « box-ticking »: les entreprises seraient dans une démarche de respect formel des dispositions plutôt que dans celle d'une adhésion réelle aux principes de gouvernance. Ainsi la transparence se transformerait en apparence et l'information diffusée ne pourrait être qu'un habile paravent. Même si la transparence est l'une des exigences d'un gouvernement d'entreprise efficace, les dirigeants seraient septiques sur l'idée qu'une meilleure gouvernance soit créatrice de valeur.

\section{Les sources d'inefficacité des mécanismes de gouvernance}

\subsection{Gouvernance et création de valeur}

Le lien entre la bonne gouvernance de la firme et la création de valeur est sujet à de nombreux débats et discussions. Les différentes études traitant du gouvernement d'entreprise mettent en relief la relation entre la gouvernance et la performance des entreprises soit d'un point de vue global, soit en s'intéressant à un mécanisme de gouvernance en particulier.

En effet, les investisseurs exigent des compagnies de mettre en application des principes rigoureux de gouvernement d'entreprise afin de réaliser de meilleures rentabilités sur leurs investissements. Gugler, Mueller et Yurtoglu (2003) confirment qu' « un système de gouvernance fiable est un système qui aligne les intérêts des dirigeants et des actionnaires et conduit à une maximisation de la richesse des actionnaires ». Campos, Newell et Wilson (2002) ont étudié le lien existant entre

132. Cette dissociation des fonctions était interdite par la loi dans la société anonyme à forme moniste et la seule solution pour dissocier les fonctions était d'avoir recours à la société anonyme à conseil de surveillance et directoire. Mais cette forme n'était adoptée qu'à moins de $4 \%$ pour l'ensemble des sociétés anonymes. 


\section{3 \\ management \& avenir}

la gouvernance et la valeur de l'entreprise et ont montré que de bonnes pratiques de gouvernance sont reconnues par une évaluation plus élevée du marché. Une firme qui investit dans les droits de l'actionnaire, dans la transparence de l'information et dans l'indépendance du conseil d'administration génère plus de confiance auprès des actionnaires. De même, Gompers, Ishii et Metrick (2003) démontrent dans leur étude une relation positive entre la bonne gouvernance et la performance de l'entreprise. En adoptant des stratégies d'investissement basées sur les meilleures pratiques de gouvernance, les investisseurs réalisent un rendement anormal de $8,5 \%$. Ils en concluent que les bonnes pratiques de gouvernance d'entreprise ont un effet considérable sur la réputation de la firme en matière de création de la valeur. Black, Jang et Kim, (2003) ont construit un indice de gouvernance d'entreprise de 526 firmes coréennes fondé sur six critères, les droits de l'actionnaire, le conseil d'administration, le processus d'audit, les directeurs indépendants, la transparence de l'information et de la structure de propriété. L'analyse de cet indice révèle une corrélation positive forte entre l'indice de gouvernance d'entreprise et la valeur de la firme. En comparant la performance des firmes de bonne gouvernance de celles qui ne le sont pas, Bai, Liu, Song et Zhang (2003) ont montré que les investisseurs sont prêts à payer une prime plus élevées pour les firmes qui adoptent les meilleures pratiques de gouvernance. De même, Drobetz, Schillhofer et Zimmermann (2003) ont construit un indice de gouvernance d'entreprise intégrant des variables comme les droits des minoritaires, le comité d'audit, la transparence, et le conseil d'administration. Le calcul de cet indice de gouvernance pour les firmes allemandes leur a permis de mettre en évidence une relation positive entre le score de gouvernance et la valeur de ces firmes allemandes. Avec un échantillon de 55 firmes françaises du SBF120, Amir (2007) confirme le rôle primordial joué par les mécanismes de gouvernance pour créer de la valeur. Selon les résultats de son étude, le bon fonctionnement du conseil d'administration lié à sa structure et son indépendance et l'existence d'un comité d'audit au sein du conseil jouent un rôle important dans la détermination de la performance des firmes françaises. En revanche, les pratiques relatives à la politique de rémunération, la structure de propriété et les droits des actionnaires ne semblent pas avoir d'effet significatif sur la performance des firmes. Ce résultat n'est pas cohérant avec les recommandations de la plupart des codes de bonnes conduites en matière de gouvernance et de création de la valeur.

Toujours dans le contexte de l'association entre la création de valeur et la gouvernance de l'entreprise, quatre mécanismes spécifiques de gouvernance sont plus particulièrement abordés dans la littérature : l'indépendance du conseil d'administration, la taille du conseil d'administration, la séparation du président et du directeur général (CEO) et la rémunération incitative.

L'indépendance des administrateurs est l'un des éléments déterminant de gouvernement d'entreprise et de son efficacité. Cependant, les études 


\section{Les sources d'inefficacité des mécanismes de gouvernance d'entreprise}

académiques relatives à l'impact des administrateurs indépendants sur la performance soutiennent des résultats controversés, essentiellement dû au fait qu'un administrateur non indépendant (interne) ne peut pas forcément soutenir la même stratégie de création de valeur qu'un administrateur indépendant (externe). Dans le contexte des structures d'actionnariat dispersées, Gagnon et St-pierre (1995) relèvent une association positive entre la présence d'administrateurs indépendants et le taux de rentabilité des capitaux des sociétés canadiennes. Agrawal et Knoeber (1996) notent une relation négative entre la présence d'administrateurs indépendants au conseil et la performance. Hermalin et Weisbach (2003) concluent qu'il existe peu de preuves, du moins aux EtatsUnis, quant à une relation entre la performance et la composition du conseil. D'autres études ont affirmé une absence d'influence des administrateurs indépendants sur la performance à l'instar de Lawrence et Stapledon (1999) ou d'Alexander et Paquerot (2000). En mesurant la performance par le Q de Tobin, André et Schiehll (2004) révèlent une relation positive entre la performance des sociétés canadiennes et la proportion d'administrateurs indépendants. Leurs résultats suggèrent qu'une augmentation moyenne de $10 \%$ de la proportion d'administrateurs indépendants conduit à une augmentation de $9 \%$ de la performance de l'entreprise.

La taille du conseil est une des autres caractéristiques du conseil d'administration fréquemment étudiées. Plusieurs auteurs prétendent que le conseil perd de son efficacité lorsqu'il devient trop grand. Bhagat et Black (2002) testent cette affirmation et la confirment ${ }^{133}$. Les conseils d'administration de petite taille sont donc synonymes d'un contrôle plus efficace sur les dirigeants. Toutefois, un conseil très restreint réduit la possibilité d'avoir une expertise diversifiée comme le montrent Daily et al. (1999).

Une autre variable étudiée est le partage des responsabilités du président du conseil d'administration (CA) et du directeur général. Les études n'ont pas trouvé les mêmes résultats concernant la relation entre la dualité (lorsque les fonctions de directeur général et président du CA sont détenues par une seule personne) et la performance. L'analyse de Dalton et al. (1998) conclut que les marchés sont plutôt indifférents à ce concept de dualité alors que Worell, Nemec et Daidson (1997) concluent à une influence négative de la dualité.

Lorsque les paniers de rémunérations des dirigeants sont étroitement liés à la performance des firmes, il est largement reconnu dans la littérature que les coûts associés aux conflits d'intérêts sont réduits ${ }^{134}$. Dans leur étude, André et Schiehll (2004) déterminent une association positive entre la performance des firmes canadiennes et l'importance de la rémunération incitative du dirigeant. Une augmentation de $10 \%$ de l'importance relative de la rémunération incitative du 
directeur général (CEO) entraine une augmentation semblable de la performance de l'entreprise. Les CEO ayant de plus grands niveaux de rémunération variable tendent à mieux aligner leurs intérêts sur ceux des actionnaires.

\subsection{La défaillance des mécanismes de gouvernance en France}

La crise économique et financière actuelle met en exergue les faiblesses de certains mécanismes de gouvernance en France. En particulier, les récents scandales entourant l'éviction ou le départ négocié des dirigeants d'entreprises cotées ont mis en évidence qu'une partie des rémunérations des dirigeants était déconnectée de leur efficacité. Précisons que la rémunération des dirigeants est déterminée par le conseil d'administration, ce dernier pouvant être appuyé par un comité de rémunération. Toutefois, ni la loi ni l'autorité des marchés financiers (AMF) n'oblige les conseils d'administration à faire appel à un comité de rémunération. Ce comité demeure un instrument purement préparatoire à la délibération du conseil d'administration. Depuis la loi TEPA du 21 août 2007, la rémunération des dirigeants de sociétés cotées est soumise à l'approbation des actionnaires et à des critères de performance. Ces critères sont l'objet d'une totale imprécision puisqu'ils ne sont ni définis, ni précisés dans les textes et sont au regard de la pratique des critères individuels liés à des objectifs financiers ou stratégiques, propres à la société considérée ${ }^{135}$. Seul le conseil d'administration sous le contrôle de l'assemblée générale détermine ces indices de performance mais cette détermination soumise à publicité peut-être par son mécanisme d'adoption totalement arbitraire et discrétionnaire. Les dirigeants peuvent ainsi bénéficier de certaines rémunérations excessives, en complète déconnexion avec la performance financière de la firme. Seules les rémunérations différées des dirigeants (indemnités de départ) sont interdites par la loi TEPA si elles ne sont pas subordonnées au respect des conditions liées aux performances du bénéficiaire. De leur côté, l'AMF et l'AFEP/MEDEF appellent également à une subordination des rémunérations différées aux performances des dirigeants, mais sans guère de précision sur cette notion de performance. Pourtant, selon une étude publiée par la Tribune, les patrons français ont perçu en 2007 les indemnités de départ les plus élevées par rapport à celles de leurs homologues européens ${ }^{136}$. Une proportionnalité entre le montant des indemnités de départ (tel que le parachute doré) et la performance réelle du dirigeant permettrait de justifier plus sereinement les engagements indemnitaires. Selon l'enquête de Hewitt Associates, en 2007, 45\% des sociétés du SBF120 ont mis en place un seuil de performance au-delà duquel le versement de l'indemnité due au titre du mandat deviendrait effectif. L'objectif visé par les entreprises à travers ce dispositif consiste à sanctionner la contre-performance des dirigeants. La question du niveau d'exigence de ce seuil est alors posée. A l'inverse, 55\% des firmes du SBF120 ont mis en place un dispositif où l'indemnité de départ croît 


\section{Les sources d'inefficacité des mécanismes de gouvernance d'entreprise}

en fonction du niveau de performance. Cette démarche parait ambiguë dans la mesure où d'autres éléments de rémunération tels que le variable annuel ou les stock options sont mieux à même de reconnaître la performance que l'indemnité de départ. II semble même qu'en France les entreprises dont les rémunérations des dirigeants ont été déconnectées des performances financières des sociétés qu'ils dirigeaient sont celles là même qui ont cédé à la tentation ou à la pression de créer des comités de rémunérations. C'est le cas de France Telecom qui s'est doté de trois nouveaux comités en 2005: le comité de rémunération, de sélection et d'organisation, le comité stratégique et le comité d'orientation. Ces trois comités sont venus s'ajouter au comité d'audit déjà existant. Certaines sociétés bien que disposant des comités spécialisés n'ont pas pu éviter les scandales. C'est le cas de Vivendi Universal sous Jean-Marie Messier ${ }^{137}$.

La création de comités de surveillance au sein des conseils d'administration est l'une des modalités d'amélioration du fonctionnement de cet organe, et plus largement de la gouvernance d'entreprise, préconisée par les codes de bonne conduite, notamment le premier rapport Viénot (1995). Toutefois, en droit français, ces comités (comité d'audit, comité de rémunération et comité de sélection) n'engagent pas leur responsabilité vis-à-vis des actionnaires et ainsi ne répondent pas à l'exigence d'indépendance véhiculée par le concept anglosaxon. Non seulement l'adoption des comités reste à la discrétion des entreprises mais ces comités ne peuvent avoir qu'un pouvoir purement consultatif. Suite à l'observation de la défaillance de ce mécanisme de gouvernance, certains auteurs (dont Pochet et Yeo, 2004) ont suggéré que la création des comités de surveillance ne revêtait en France qu'un caractère purement formel, destiné à satisfaire aux exigences des investisseurs anglo-saxons, sans traduire une réalité de fonctionnement répondant aux attentes du marché, spécialement en matière d'indépendance vis-à-vis du dirigeant. Dés lors, se pose la question de savoir ce qui motive leur adoption par un nombre toujours croissant d'entreprises cotées.

Outre l'indépendance des comités de surveillance, celle des administrateurs est également compromise et ceci pour deux raisons principales : les intérêts financiers minimes dont ils bénéficient ${ }^{138}$ et leur nomination souvent appuyée par le directeur général. Particulièrement en France, la nomination des administrateurs a toujours été considérée par les dirigeants comme une prérogative personnelle. C'est pourquoi, même lorsqu'un comité de sélection a été mis en place, les dirigeants et plus particulièrement le directeur général continuent à influencer fortement le processus de sélection. Au demeurant, le fonctionnement du conseil d'administration peut être affecté par l'existence de liens humains qu'il entretient

137. Jean-Marie Messier quittait Vivendi en juillet 2002 et laissait une entreprise au bord de la faillite. Son dernier salaire d'un montant de 5,6 millions d'euros marquait une augmentation de 10\% par rapport à l'année précédente. Dans le même temps, Vivendi accusait une perte nette de 23 milliards d'euros. En démissionnant, Jean-Marie Messier réclamait 20,5 millions supplémentaires comme parachute doré.

138. Les administrateurs ne trouvent pas dans leur rémunération la juste reconnaissance des risques encourus. 


\section{3 \\ management \& avenir}

avec d'autres conseils. Plusieurs études révèlent la présence de réseaux d'administrateurs ou de relations bilatérales entre administrateurs au sein des sociétés du CAC 40 ${ }^{139}$. Pour la théorie de l'enracinement, ce sont les réseaux d'administrateurs indépendants " amis » qui siègent dans les conseils et comités spécialisés. Leurs recrutements et affectations ne sont plus associés à leurs compétences, ce qui réduit l'intérêt de les soumettre à une quelconque évaluation. Selon certains auteurs ${ }^{140}$, un administrateur qui appartient au même cercle social (polytechniciens ou énarques) que celui du dirigeant verra sa probabilité plus élevée d'obtenir un mandat. L'existence de réseaux d'administrateurs remet en cause leur indépendance et se traduit par une certaine inefficacité du contrôle interne. Ainsi, les réseaux d'administrateurs ont une image négative dans l'opinion publique en France dans la mesure où ils sont supposés avoir une influence néfaste sur la performance des firmes ${ }^{141}$. Les mêmes tendances ont été observées pour les dirigeants des grandes entreprises cotées. Les dirigeants grands corps ne sont pas réellement sur un marché du travail et semblent quitter plus difficilement leur emploi lorsqu'ils côtoient au moins un administrateur du même cercle social (énarques ou grands corps) : leur révocation est moins probable en cas de mauvaise performance et, s'ils sont révoqués, ils tendent à retrouver un autre emploi au moins aussi avantageux que le précédent. Autre observation tout aussi inquiétante, le réseau des énarques s'est approprié le pouvoir dans de grands établissements financiers, ce qui génère un avantage concurrentiel aux dirigeants d'entreprises issus de la même grande école en termes de politique d'endettement par rapport à leurs homologues polytechniciens ${ }^{142}$.

Une autre défaillance des mécanismes de gouvernance observée par l'Institut Français des Administrateurs (IFA) relève de la relation administrateur-actionnaire qui se limite souvent aux discussions à l'assemblée générale et encore quand les administrateurs sont présents. Pour tenter de remédier à cette défaillance, I'IFA a procédé, en mai 2007, à des propositions pour une meilleure relation entre les administrateurs et les actionnaires. Ces propositions se traduisent non seulement par la prise en compte de l'intérêt de l'actionnaire dans le fonctionnement du conseil d'administration mais également par l'implication des administrateurs dans la communication entre la société émettrice et ses actionnaires en dehors de l'assemblée générale.

\section{Conclusion}

Dans les grandes sociétés cotées, la gouvernance est avant tout perçue comme un moyen de contrôle, plus que comme un outil d'accompagnement des grandes orientations stratégiques. Les évolutions des mécanismes de gouvernance ont été telles ces dernières années que certaines sociétés cotées n’hésitent plus à

139. Se référer à Chabi et Maati $(2005,2006)$ pour leurs études sur les sociétés du CAC40 de 1996 à 2004.

140. Voir Kramarz et Thesmard (2006).

141. Se référer à Maati (2008)

142. Voir Nguyen-Dang (2006) et Paquerot et Chapuis (2006), pour une discussion sur ce thème. 


\section{Les sources d'inefficacité des mécanismes de gouvernance d'entreprise}

se retirer de la bourse pour éviter trop de contraintes liées aux réglementations mises en place pour protéger les actionnaires minoritaires, et qu'il est parfois difficile pour les firmes de recruter les administrateurs dont elles auraient besoin. Dés lors, les administrateurs doivent mettre en place des règles de gouvernance adaptées à leur société et veiller à ce que l'application de ces règles ne se résume pas à un exercice formel mais au contraire donne lieu à un ancrage des principes de « bonne gouvernance » dans la culture de l'entreprise.

Plusieurs dysfonctionnements observés au sein de diverses entreprises ont démontré que les mécanismes de contrôle des activités des entreprises et donc de gouvernance actuels sont remis en cause: l'existence de rémunérations excessives en complète déconnexion avec la performance des firmes, le manque de transparence de l'information délivrée aux actionnaires concernant les critères de performance retenus pour positionner l'entreprise et déterminer la rémunération des dirigeants, l'accroissement et la médiatisation des scandales impliquant les dirigeants ; le manque de clarté dans le fonctionnement des conseils d'administration. Ainsi, il est important pour les entreprises d'améliorer leurs pratiques en matière de gouvernance afin que les actionnaires soient plus confiants. Mais restons vigilants, la gouvernance n'est pas une assurance. La plus intègre des firmes ne garantit ni la pertinence des choix stratégiques, ni la croissance des résultats, ni même l'absence de dérives.

\section{Bibliographie}

Agrawal A. et Knoeber C.R., (1996), "Firm Performance and Mechanisms to Control Agency Problems Between Managers and Shareholders", Journal of Financial and Quantitative Analysis, September, p 377-397.

Aguilera R. et Cuervo-Cazurra A., (2004), « Codes of Good Governance Worldwide : What is the Trigger? », Organization Studies, vol.25, $n^{\circ} 3, \mathrm{p} 415-443$.

Alexander H. \& Paquerot M., (2000), "Efficacité des structures de contrôle et enracinement des dirigeants », Finance, Contrôle, Stratégie, Vol.3, n², p 5-29.

Amir L., (2007), "Les pratiques de gouvernance et a performance des entreprises françaises", Working paper, XVlème Conférence Internationale de Management Stratégique, Montréal, 6-9 juin.

André P. et Schiehll E., (2004), « systèmes de gouvernance, actionnaires dominants et performance future des entreprises », Finance Contrôle Stratégie, Vol. 7, n², p 165-193. Bhagat S. et Black B., (1999), « The Uncertain Relationship between Board Composition and Firm Performance », The Business Lawyer, May, vol. 54, n³, p 921-963.

Bhagat S. et Black B., (2002), « The Non-correlation between Board Independence and Long Term Performance », Journal of Corporation Law, Winter, vol. 27, n², p 231-273.

Bai C.E., Liu Q., Lu J., Song F.M. et Zhang J., (2003), « Corporate governance and markets valuation in China », Journal of Comparative Economics, Vol. 32, n4, p 599-825.

Barkema H.G. et Gomez-Mejia L.R., (1998), "Managerial Remuneration and Firm Performance: A General Research Framework", Academy of Management Journal, April, vol. 41, n², p 135-145.

Black B., Jang H. and Kim W., (2003), "Does Corporate Governance Affect Firms' Market Values? Evidence from Korea", Working paper 237, Stanford Law School, John M. Olin Program in Law and Economics. 
Campos C.E., Newell R.E. and Wilson G., (2002), "Corporate governance develops in emerging markets", McKinsey on finance, p 15-18.

Chabi S. et Maati J., (2005), « les réseaux du CAC 40 », La revue du financier, n¹53, p 45-62.

Chabi S. et Maati J., (2006), « The Small Worl of the CAC 40 », Banque et Marchés, n82, p 41-53.

Charreaux G., (1991), "Structure de propriété, relation d'agence et performance financière", Revue Economique, Vol. 42, n³, p 521-552.

Charreaux G., (1997), "Vers une théorie du gouvernement des entreprises", in G. Charreaux, Le gouvernement des entreprises, Economica, p 121-164.

Core J.E., Holthausen R.W. et Larker D., (1999), « Corporate Governance, Chief Executive Officer Remuneration, and Firm Performance", Journal of Financial Economics, March, Vol.51, n³, p 371-406.

Daily C.M., Dalton D.R., Johnson \& Ellshand, (1999), « Number of Directors and Financial Performance : A meta-analysis", Academy of Management Journal, Vol.42.

Dalton D.R., Daily C.M., Ellstrand A.E. et Johnson J.L., (1998), "Meta-analytic Reviews of Board Composition, Leadership Structure, and Financial Performance", Strategic Management Journal, vol.19, n³, March, p 269-290.

Drobetz W., Schillhofer A., and Zimmermann H., (2003), "Corporate governance and expected stock return : Evidence from Germany", Working Paper, http://ssrn.com/ abstract_id=369100.

Gagnon J.M. et St-Pierre J., (1995), « Alternative Mechanisms for Corporate Governance and Board Composition », in J.D. Ronald et R. Morck, Coporate Decision-Making in Canada, University of Calgary Press, p 149-188.

Gompers P., Ishi J.L. et Andrew M., (2003), « Corporate governance and equity prices", Quarterly Journal of Economics.

Gugler K., Mueller D.C. et Yurtoglu B.B., (2003), « Corporate governance and the return on investment », Working paper, http://ssrn.com/abstract_id=299520.

Hermalin B.E. et Weisbach M.S. (1991), "The Effect of Board Composition and Direct Incentives on Firm Performance", Financial Management, vol.20, n4, p 101-112.

Jensen M. et Meckling W., (1976), "The theory of the firm : Managerial Behaviour, Agency Costs and Ownership Structure”, Journal of Financial Economics, vol. 3, p 305-360.

Kramarz F. et Thesmar D., (2006), « Social Networks in the boardroom », discussion paper 5496, CEPR.

Lawrence J. \& Stapledon G.P., (1999), "Is board compensation important? A study of listed Australian firms", Working paper, University of Melbourne.

Maati j., (2008), "Réseaux d'administrateurs et performance financière des grandes entreprises en France : une revue de la littérature », Revue française de gouvernance d'entreprises, $\mathrm{n}^{\circ} 3, \mathrm{p}$ 145-161.

McKinsey and Compagny, (2002), "Global Investor Opinion Survey : Key findings", London (http://www.mckinsey.com/governance).

Nguyen-Dang B., (2006), « Does the Rolodex Matter ? Corporate Elite's Small World and Effectiveness of Boards of Director », Conference EFA.

Paquerot M. et Chapuis J-M., (2006), "Réseaux d'appartenance des dirigeants et structures financières de leurs entreprises », conférence AFFI.

Pochet C et Yeo H., (2004), « Les comités spécialisés des entreprises françaises cotées : mécanismes de gouvernance ou simples dispositifs esthétiques ? », Comptabilité, Contrôle, Audit, vol. 2, n¹0, p 31-54.

Siala F., (2007), « Interaction des mécanismes internes de gouvernement d'entreprise et effet sur la performance », Revue française de gouvernance d'entreprises, n², p 59-82.

Stuart S., (2007), « Président directeur général ou président et directeur général », Revue française de gouvernance d'entreprises, $n^{\circ} 1$, p 27-34. 


\section{Les sources d'inefficacité des mécanismes de gouvernance d'entreprise}

Tchotourian I., (2007), « Une approche comparée de l'encadrement normatif de la rémunération des dirigeants de sociétés cotées ou la définition de la bonne gouvernance par le droit », Revue française de gouvernance d'entreprises, $n^{\circ} 2, \mathrm{p}$ 39-55.

Thiery-Dubuisson S., (2002), « Exigences actionnariales et réseaux d'administrateurs : à quoi répond la mise en place des comités d'audit en France ? », Comptabilité, Contrôle, Audit, vol. 1, n8, p129-150.

Wirtz P. (2004), « Meilleures pratiques de gouvernance, théorie de la firme et modèles de création de valeur : une appréciation critique des codes de bonne conduite », cahier du FARGO $n^{\circ} 1040401$.

Worell D.L., Nemec C. \& Davidson W.N., (1997), "One hat too many: key executive plurality and shareholder wealth", Strategic Management Journal, Vol.18, n6, p 499-507.

Yermack D., (1997), "Higher market valuation of companies with a small board of directors", Journal of financial Economics, Vol.40, p 185-211 